Supporting Information for

\title{
Molecular Linking Stabilizes Bi Nanoparticles for Efficient Electrochemical Carbon Dioxide Reduction
}

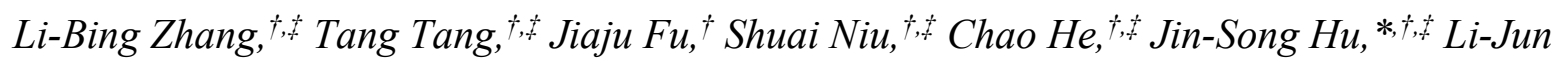
Wan. $*$, H

$\dagger$ Beijing National Laboratory for Molecular Sciences (BNLMS), CAS Key Laboratory of Molecular Nanostructure and Nanotechnology, Institute of Chemistry, Chinese Academy of Sciences (CAS), Beijing 100190, China

University of the Chinese Academy of Sciences, Beijing 100049, China

\section{Corresponding Author:}

Jin-Song Hu*: hujs@iccas.ac.cn; Li-Jun Wan*: wanlijun@iccas.ac.cn

Contents:

This file includes Figure S1-S20 and Table S1-S2. 


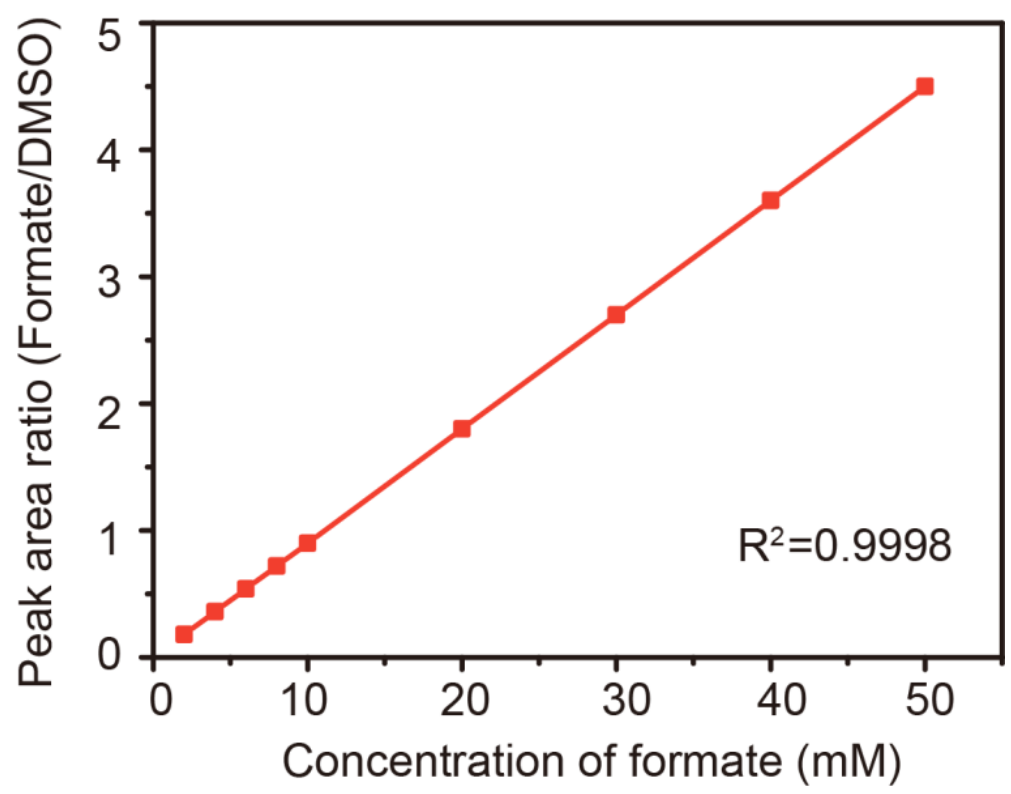

Figure S1. The calibration curve for formate with different concentrations.
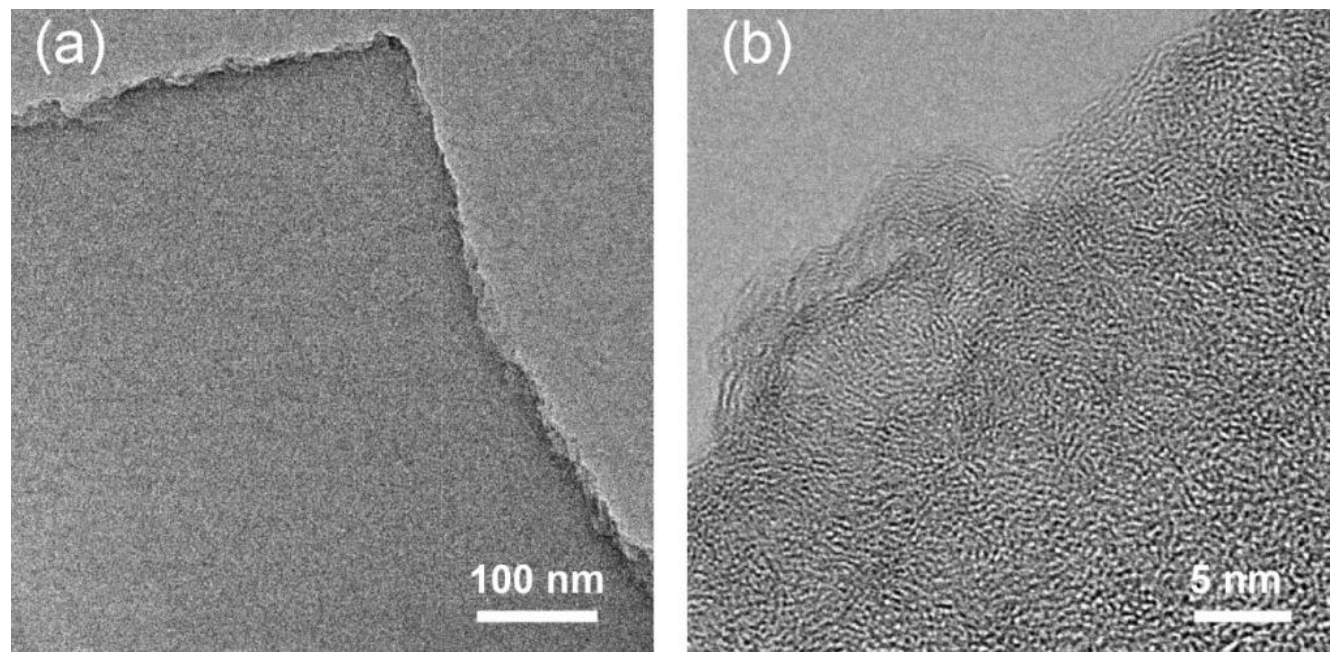

Figure S2. (a) TEM and (b) HRTEM images of the PC, showing many spots in brighter contrast which is a typical feature of nanoporous structure. 
(a)

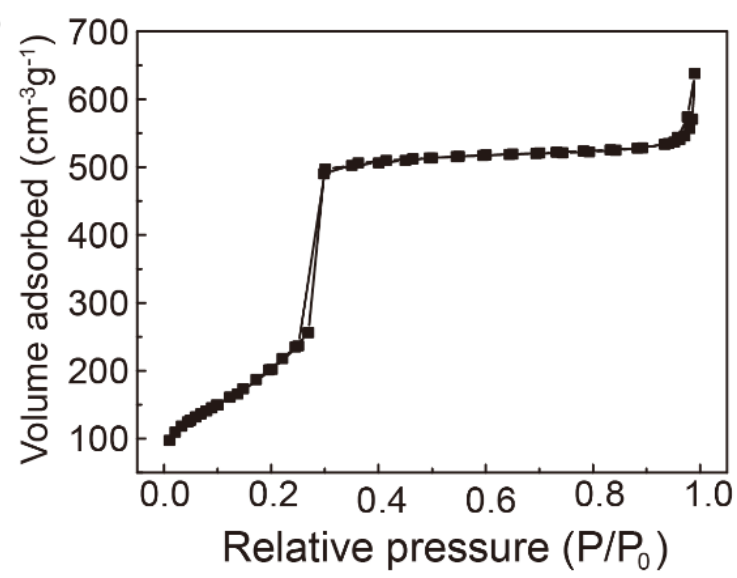

(b)

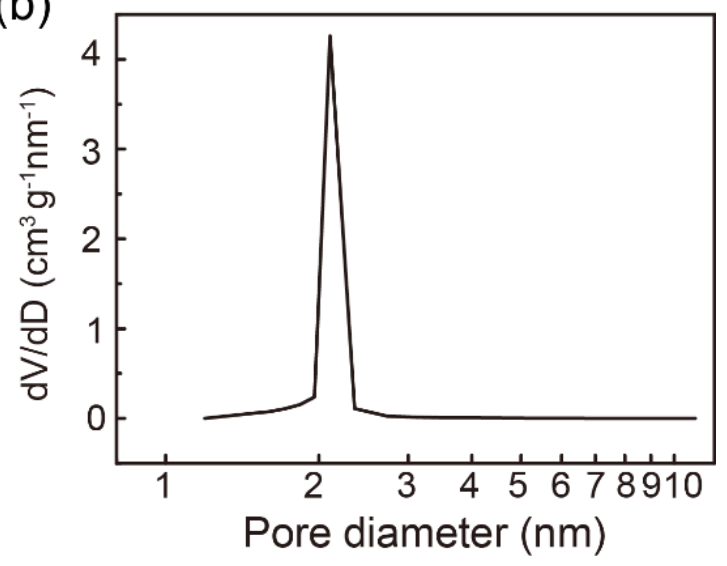

Figure S3. (a) $\mathrm{N}_{2}$ adsorption-desorption isotherms, and (b) corresponding pore size distribution curve of PC.
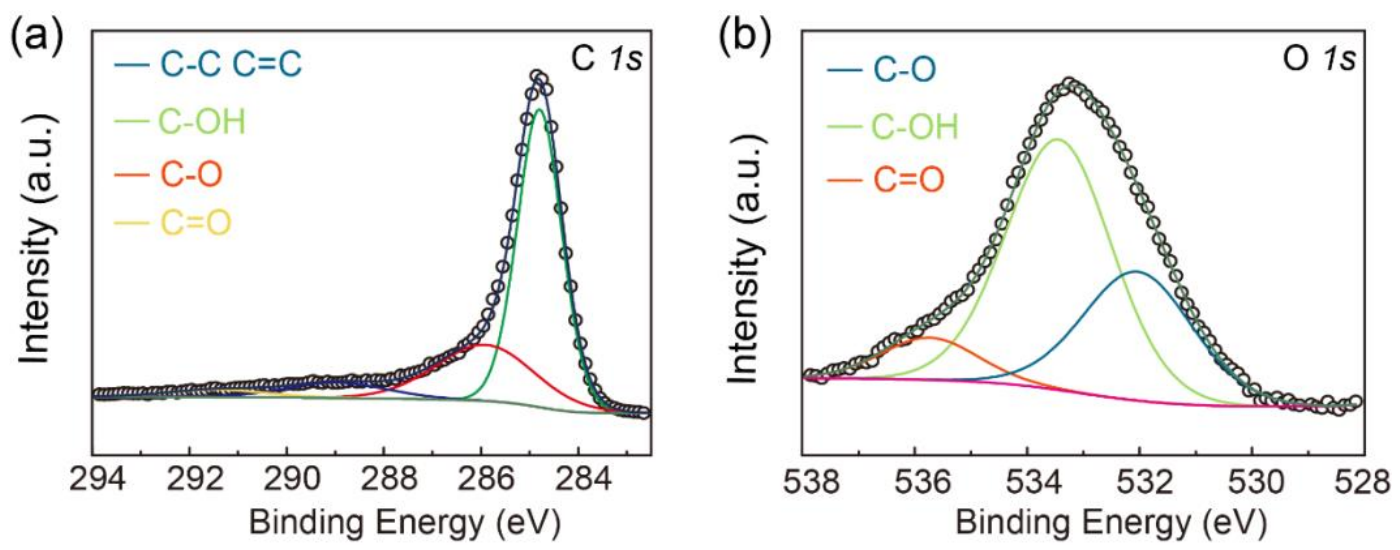

Figure S4. High-resolution XPS spectra of PC: C $1 s$ (a) and O $1 s(\mathrm{~b})$. 


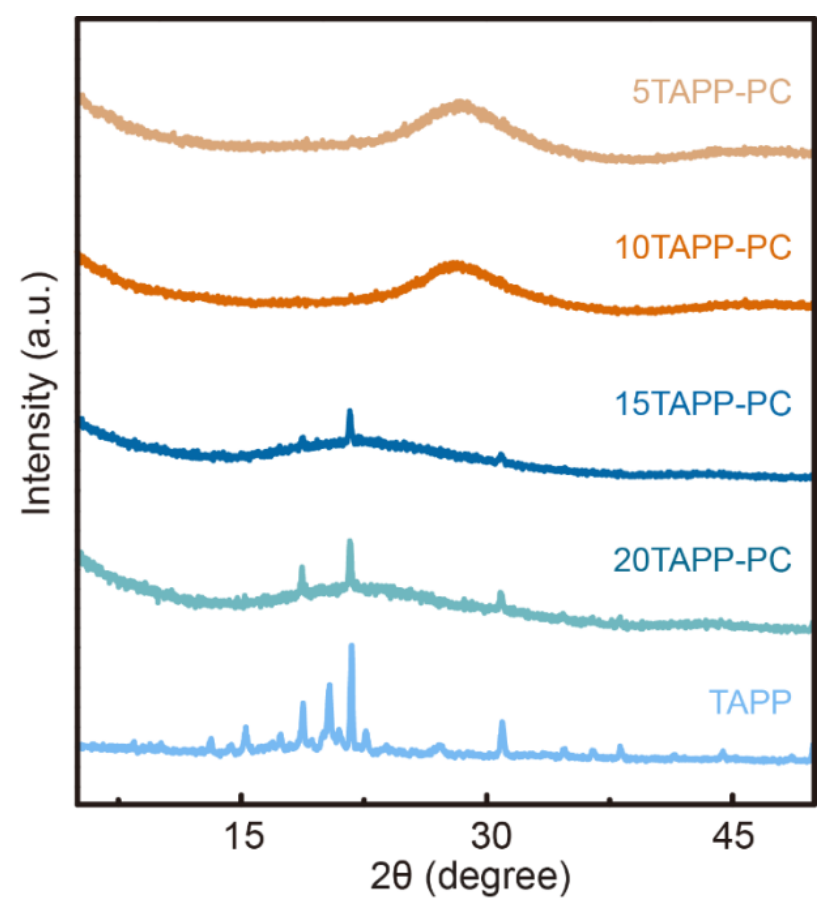

Figure S5. XRD patterns for TAPP-PCs with various TAPP loading (from 5 to $20 \mathrm{mg}$ per $60 \mathrm{mg}$ PC).
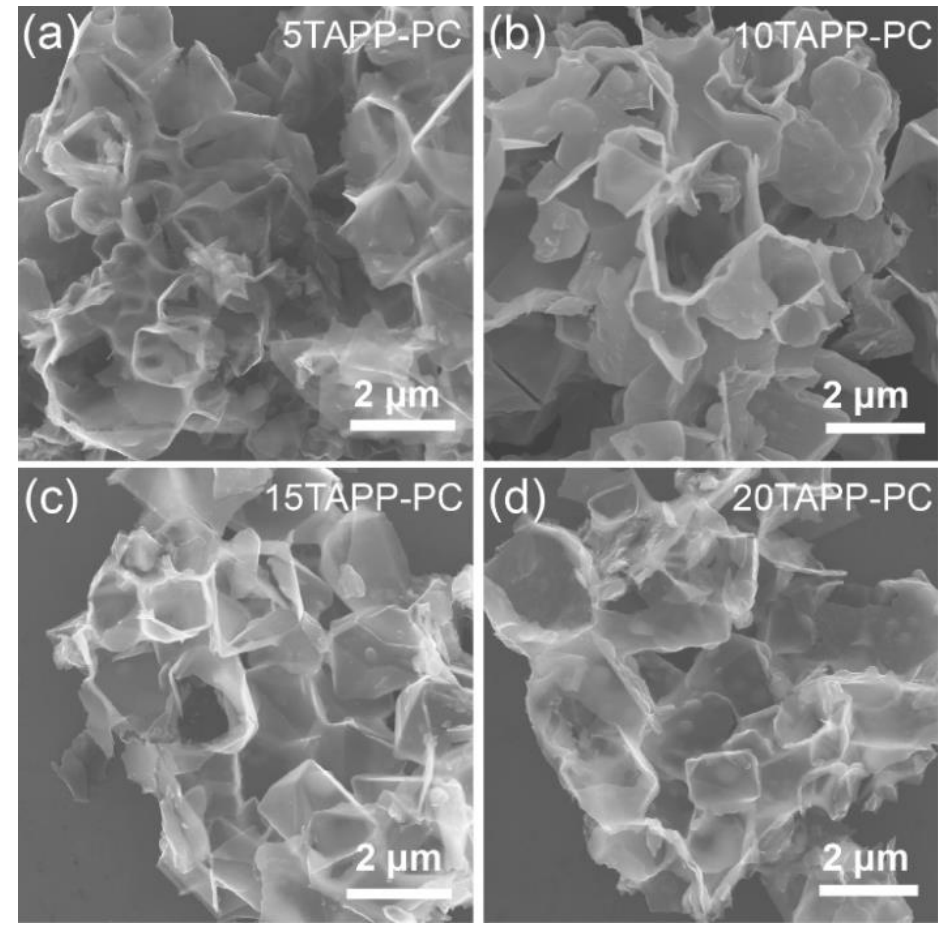

Figure S6. SEM images for TAPP-PCs with various TAPP loadings. 


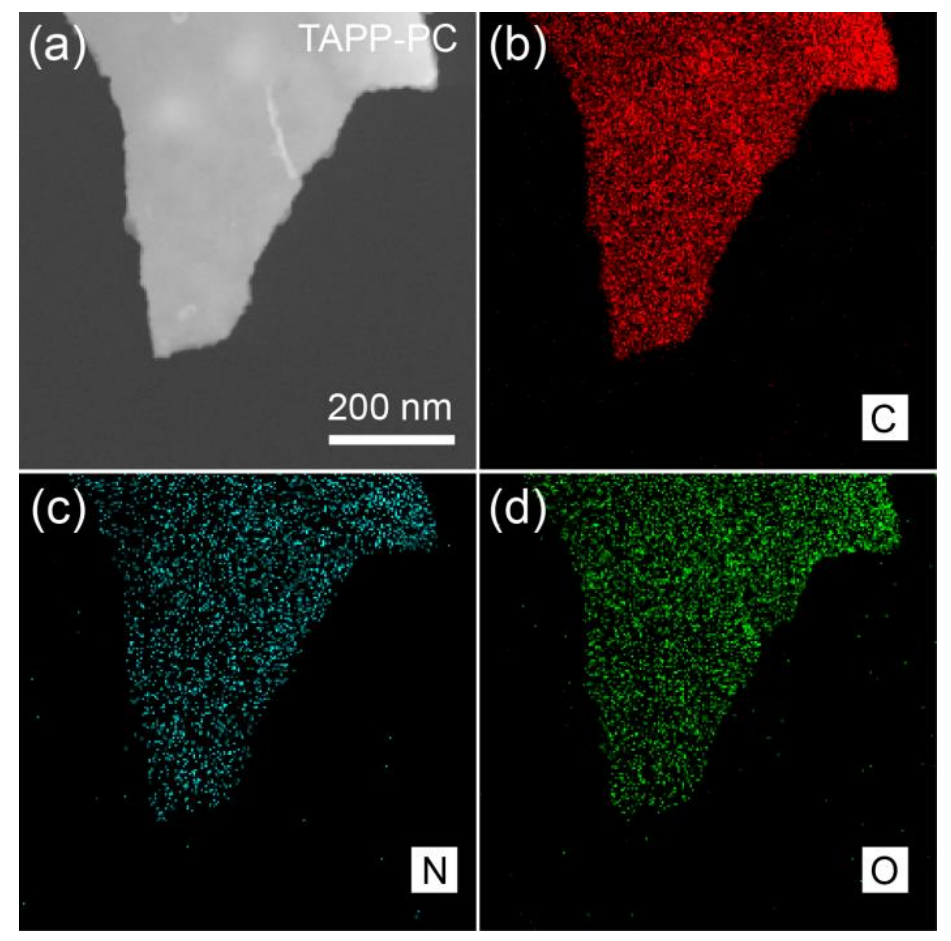

Figure S7. (a) STEM image of TAPP-PC. Corresponding EDS mapping images of TAPP-PC: (b) C, (c) $\mathrm{N}$ and (d) O.

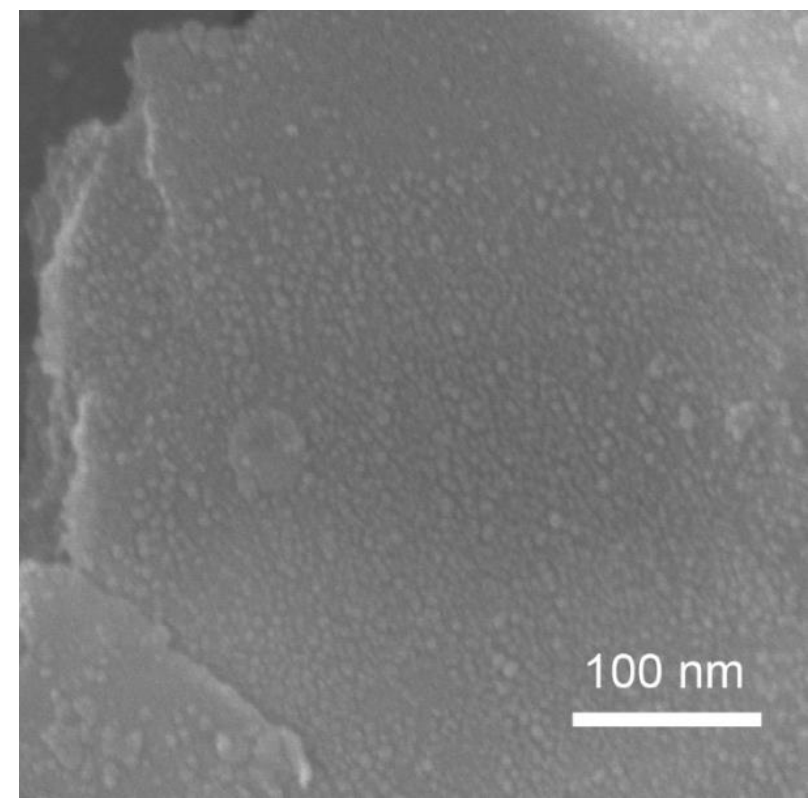

Figure S8. Low-magnification SEM image of Bi NPs/TAPP-PC. 


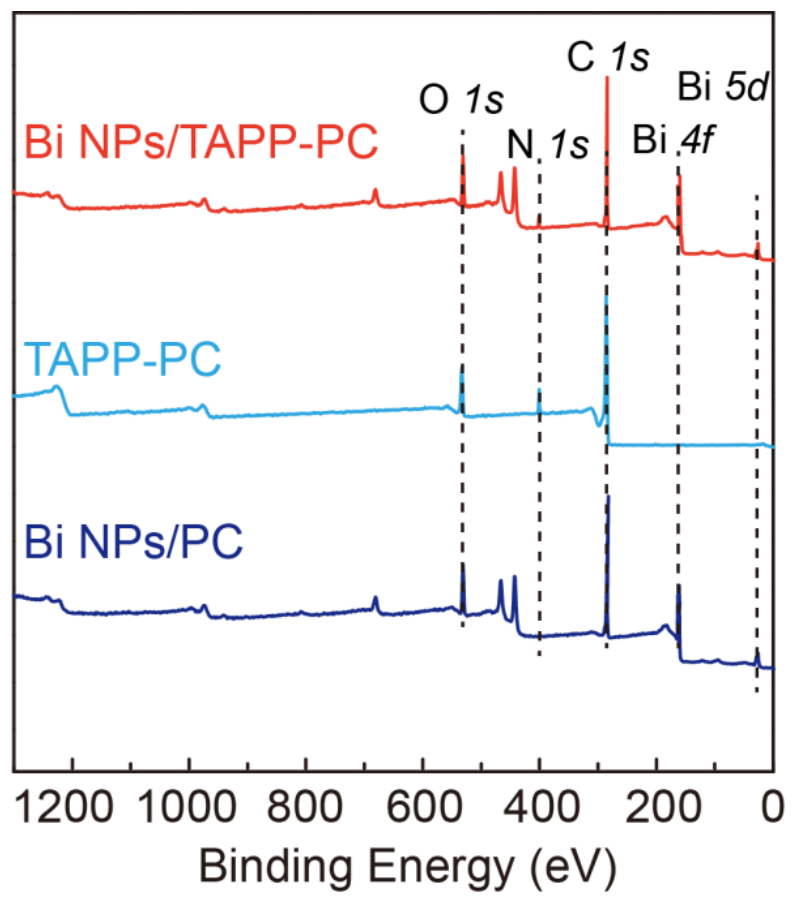

Figure S9. XPS spectra of Bi NPs/PC, TAPP-PC, and Bi NPs/TAPP-PC.
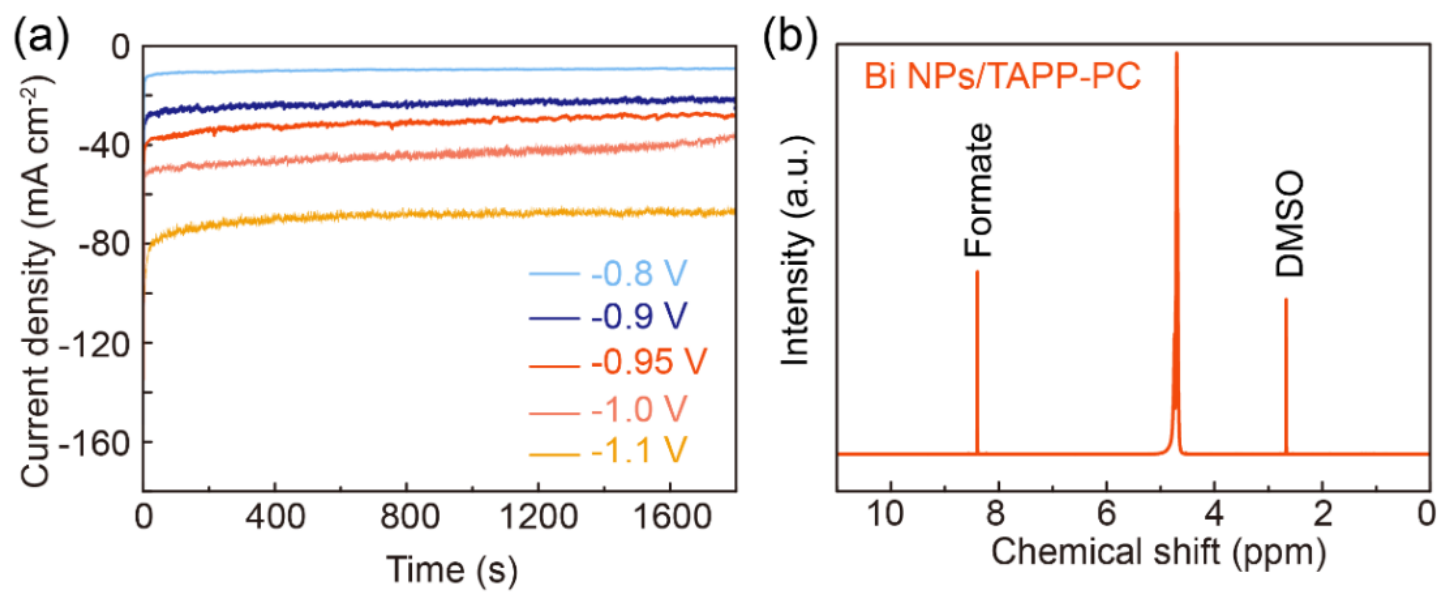

Figure S10. (a) Chronoamperometry results at each given potential in $\mathrm{CO}_{2}$-saturated $0.5 \mathrm{M}$ $\mathrm{KHCO}_{3}$ solution over Bi NPs/TAPP-PC. (b) NMR spectrum of the cathodic electrolyte after $0.5 \mathrm{~h}$ of $\mathrm{CO}_{2}$ reduction at $-0.95 \mathrm{~V}$. 

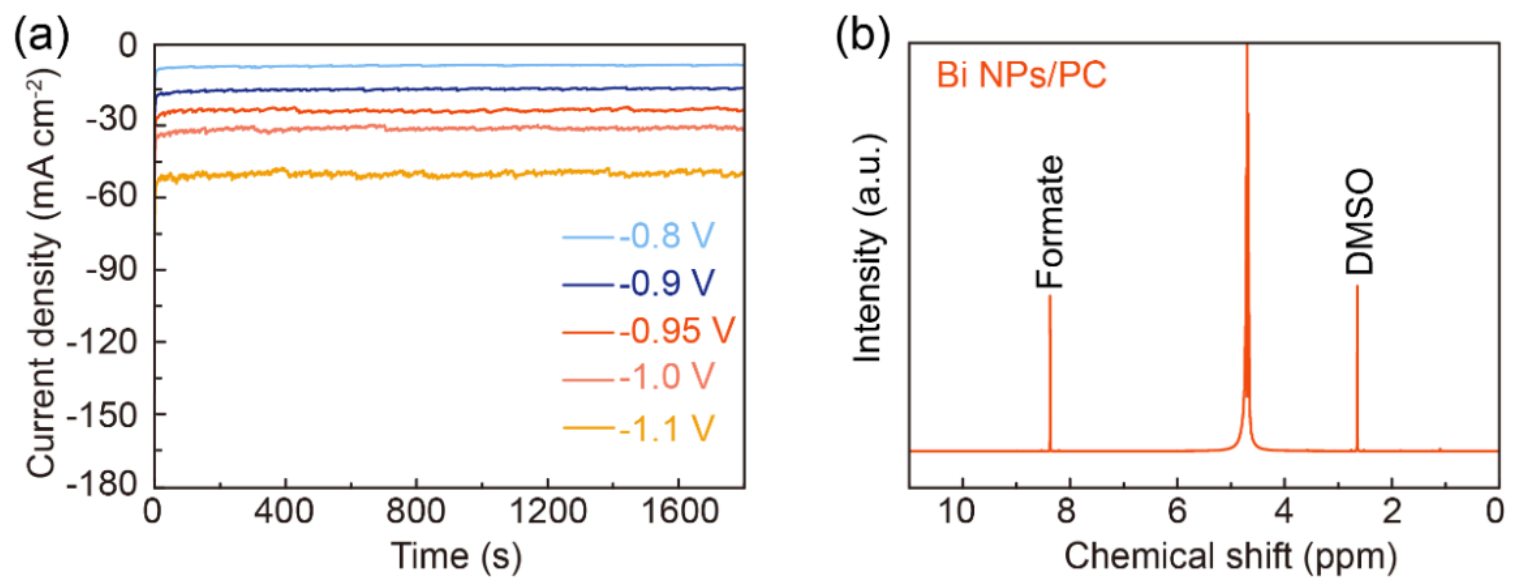

Figure S11. (a) Chronoamperometry results at each given potential in $\mathrm{CO}_{2}$-saturated $0.5 \mathrm{M}$ $\mathrm{KHCO}_{3}$ solution over Bi NPs/PC. (b) NMR spectrum of the cathodic electrolyte after $0.5 \mathrm{~h}$ of $\mathrm{CO}_{2}$ reduction at $-0.95 \mathrm{~V}$.
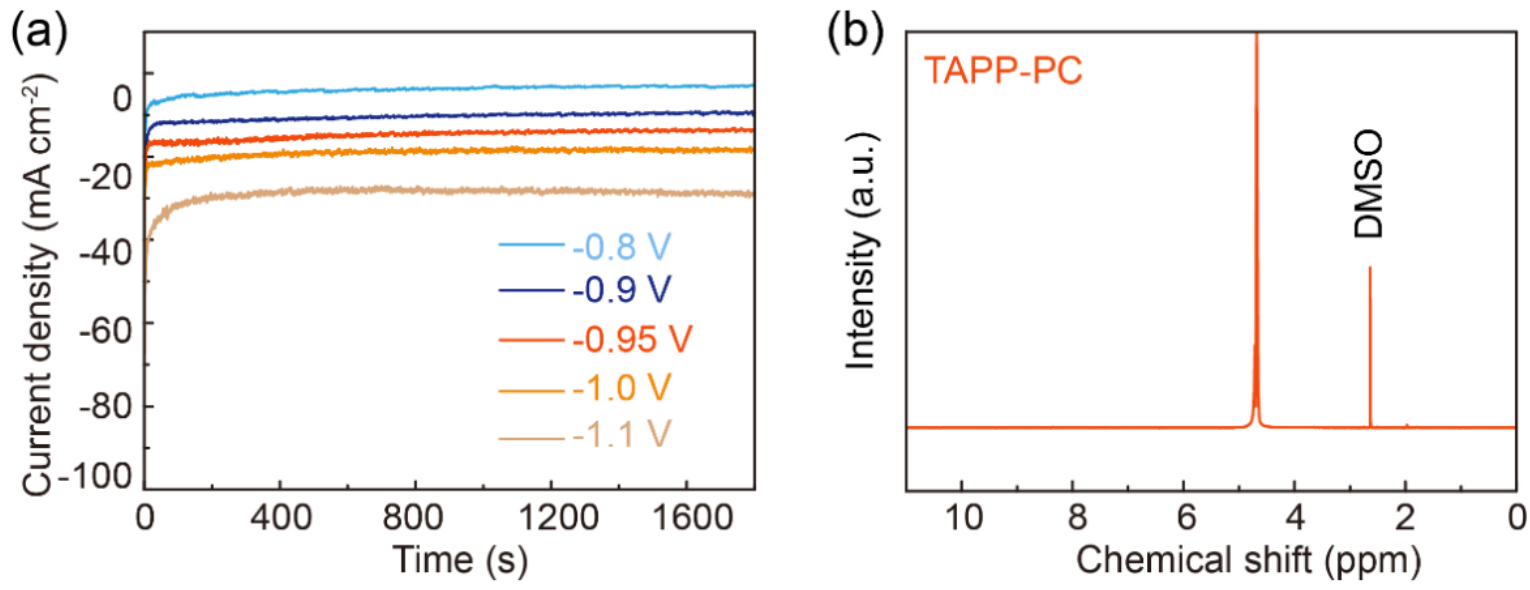

Figure S12. (a) Chronoamperometry results at each given potential in $\mathrm{CO}_{2}$-saturated $0.5 \mathrm{M}$ $\mathrm{KHCO}_{3}$ solution over TAPP-PC. (b) NMR spectrum of the cathodic electrolyte after $0.5 \mathrm{~h}$ of $\mathrm{CO}_{2}$ reduction at $-0.95 \mathrm{~V}$. 

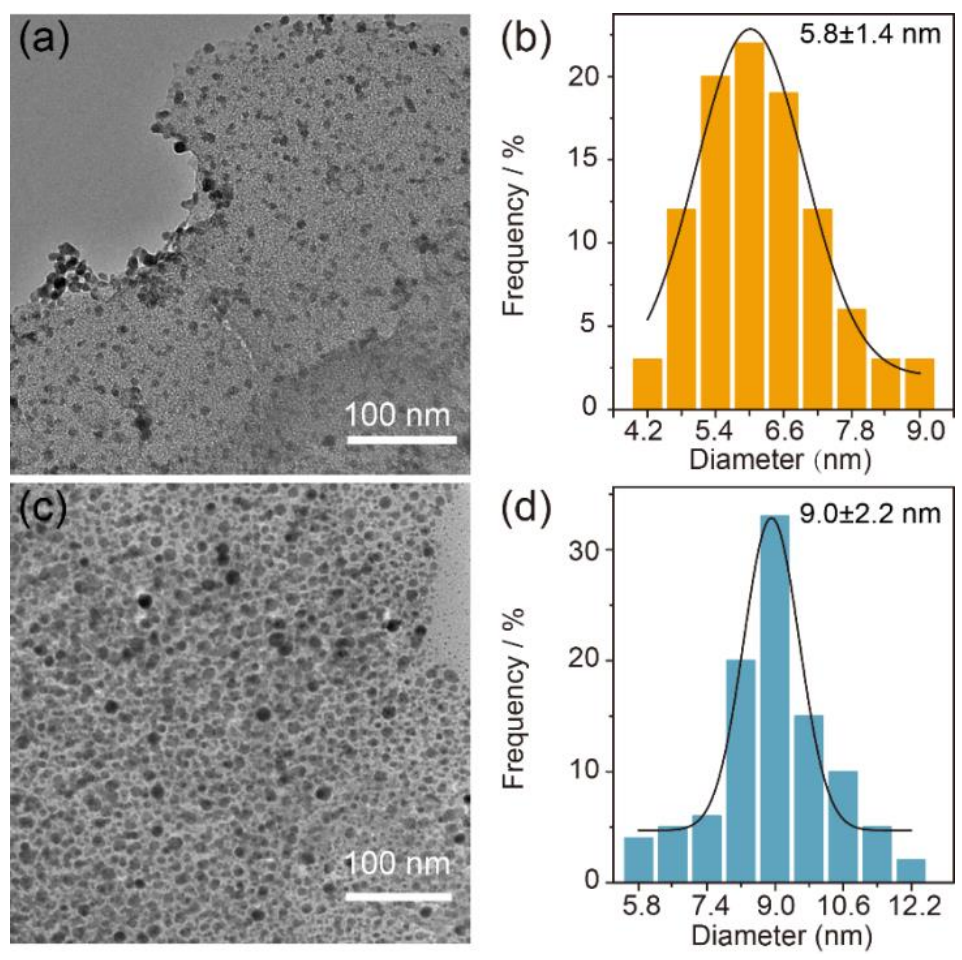

Figure S13. TEM and size distributions of Bi NPs/TAPP-PC with (a-b) lower feeding amount of bismuth nitrate $(0.1 \mathrm{mM})$ and higher feeding amount of bismuth nitrate $(1 \mathrm{mM})$.
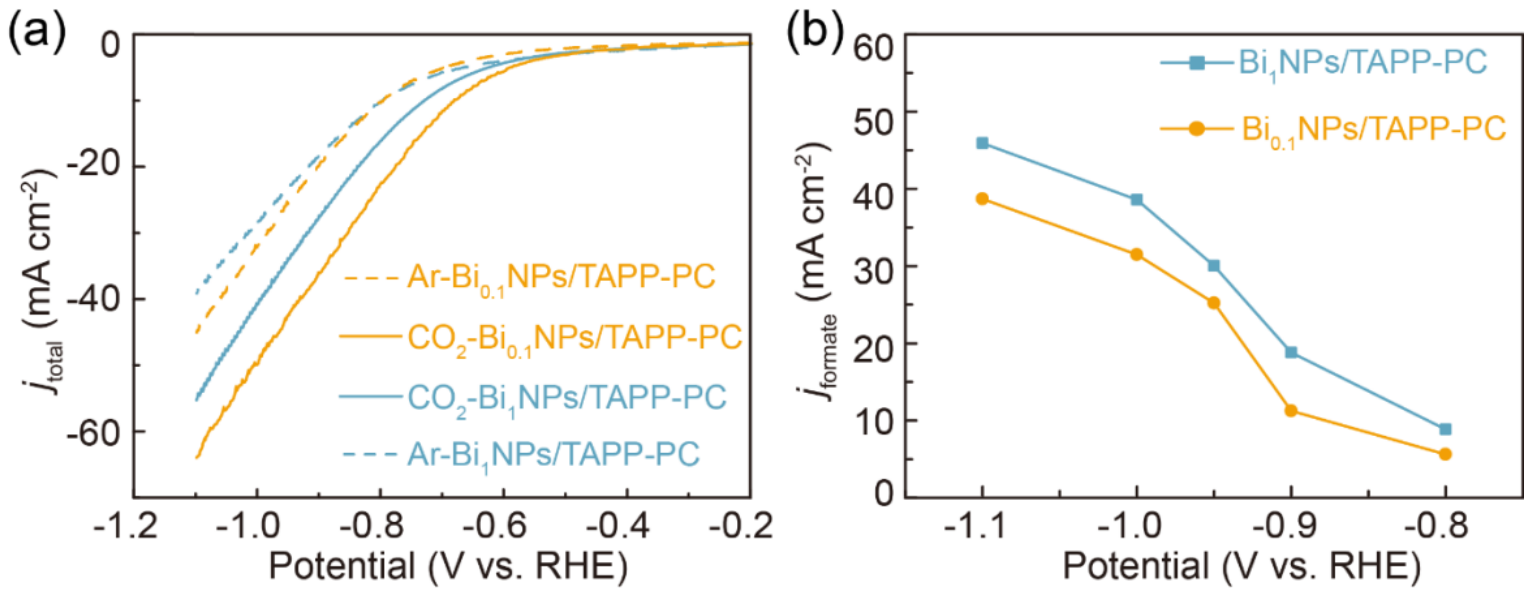

Figure S14. $\mathrm{CO}_{2} \mathrm{RR}$ performance of $\mathrm{Bi}$ NPs/TAPP-PC for different feeding amounts of bismuth nitrate. (a) LSV curves at $20 \mathrm{mV} \mathrm{s}^{-1}$ in $\mathrm{CO}_{2}$-saturated or Ar-saturated $0.5 \mathrm{M} \mathrm{KHCO}_{3}$ solution. (b) Partial current density of formate at the given potentials. 


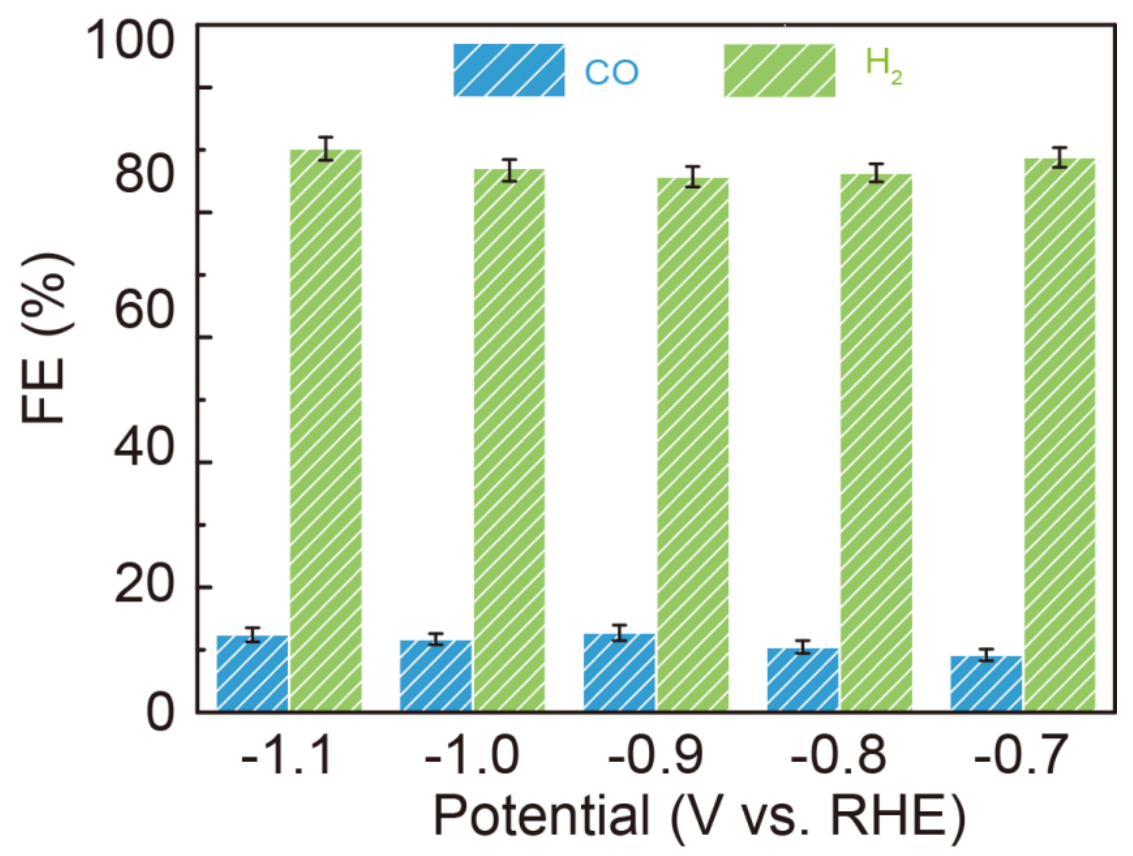

Figure S15. FEs of $\mathrm{CO}$ and $\mathrm{H}_{2}$ on TAPP-PC at the given potentials.
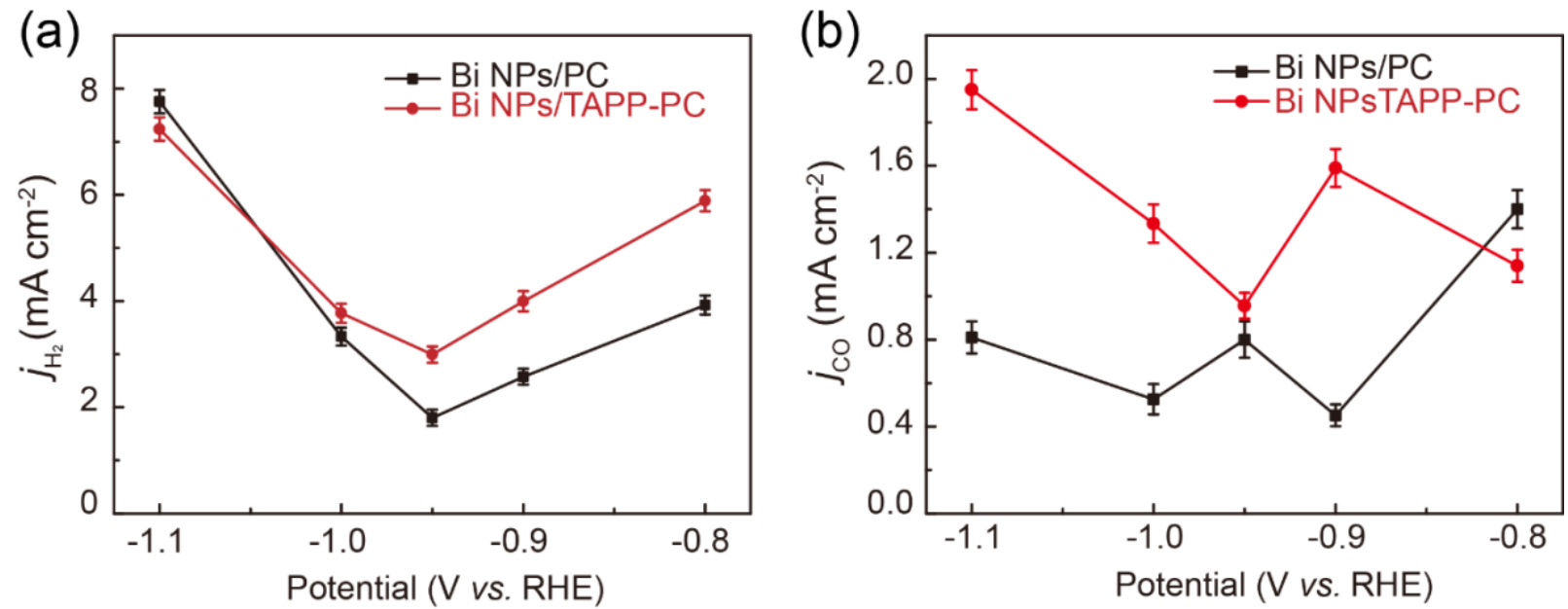

Figure S16. Partial current density of (a) $\mathrm{H}_{2}$ and (b) $\mathrm{CO}$ at the given potentials over Bi NPs/PC and $\mathrm{Bi}$ NPs/TAPP-PC. 
(a)

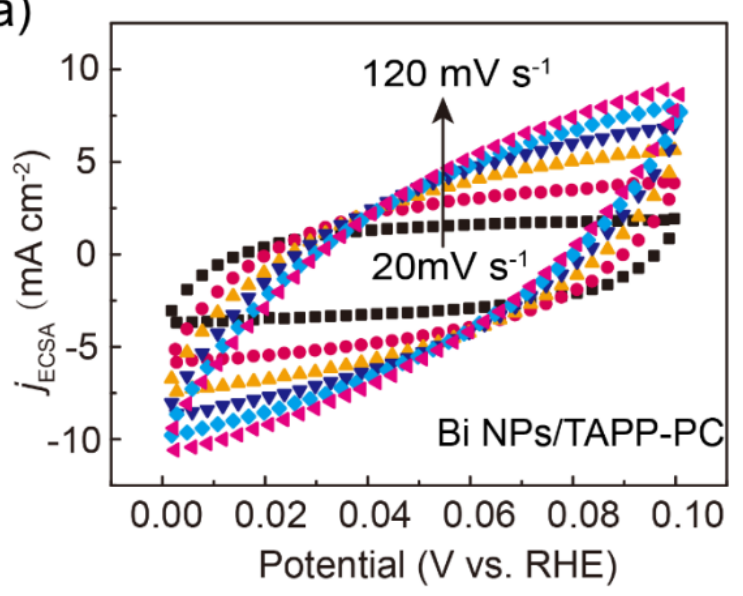

(b)

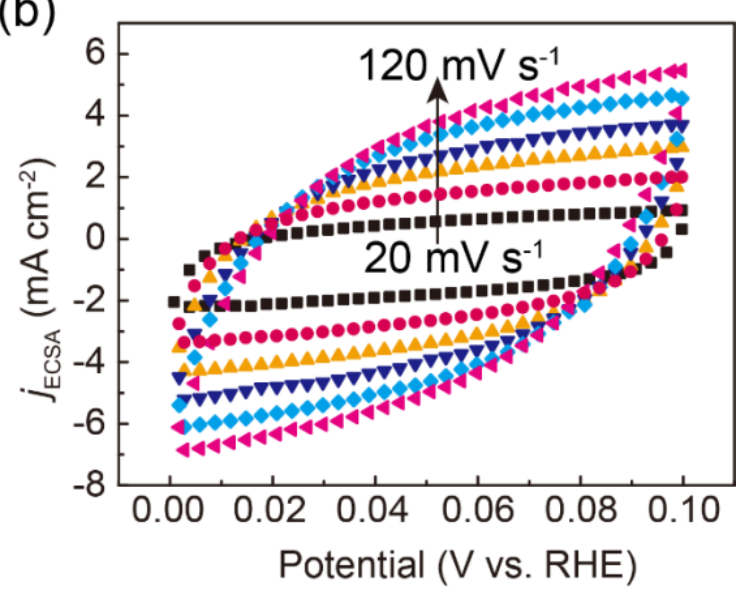

Figure S17. (a-b) CV measurements for evaluating electrochemical active surface area (ECSA) of Bi NPs/TAPP-PC and Bi NPs/PC.
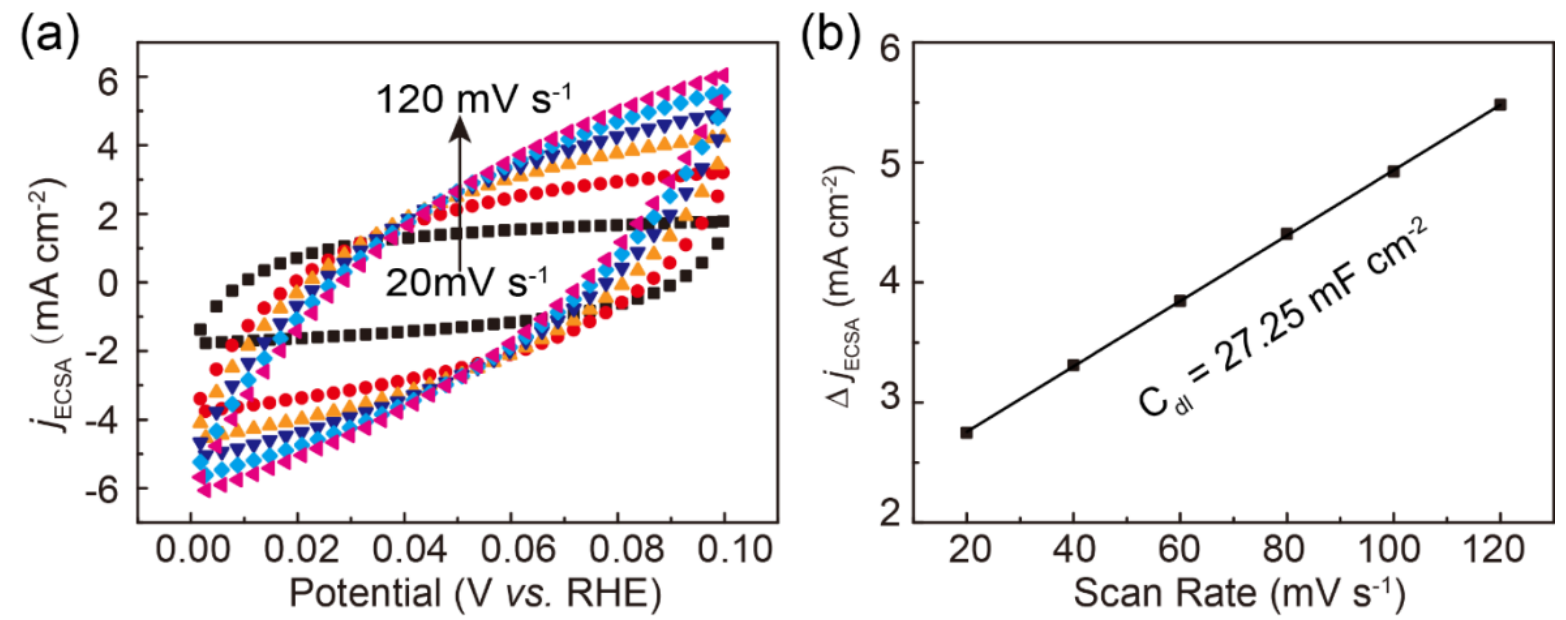

Figure S18. (a) CV measurements for evaluating electrochemical active surface area (ECSA) of TAPP-PC, (b) Capacitive currents determined at $0.05 \mathrm{~V}$ by plotting as a function of scan rates. 


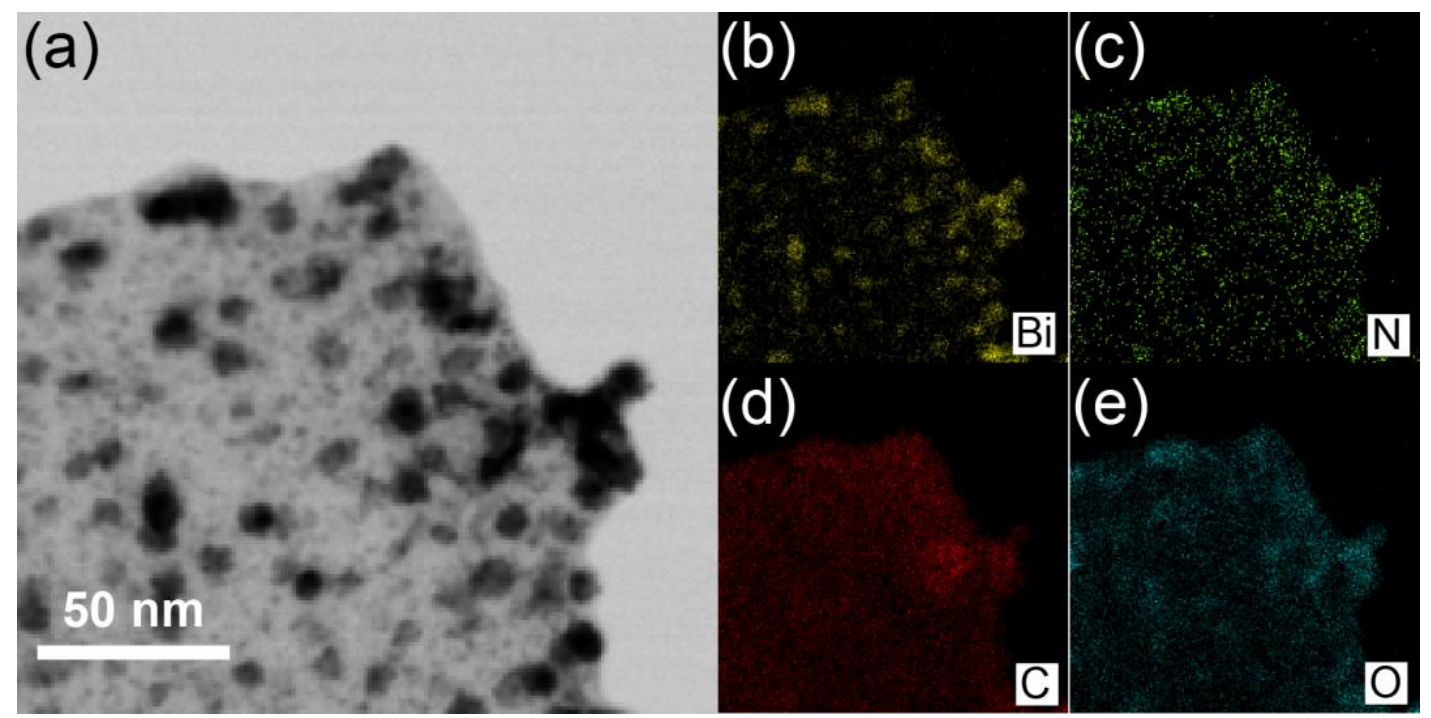

Figure S19. (a) STEM image of Bi NPs/TAPP-PC after stability test. (b-e) Corresponding EDS mapping images of TAPP-PC: (b) Bi, (c) N, (d) C, (e) O.
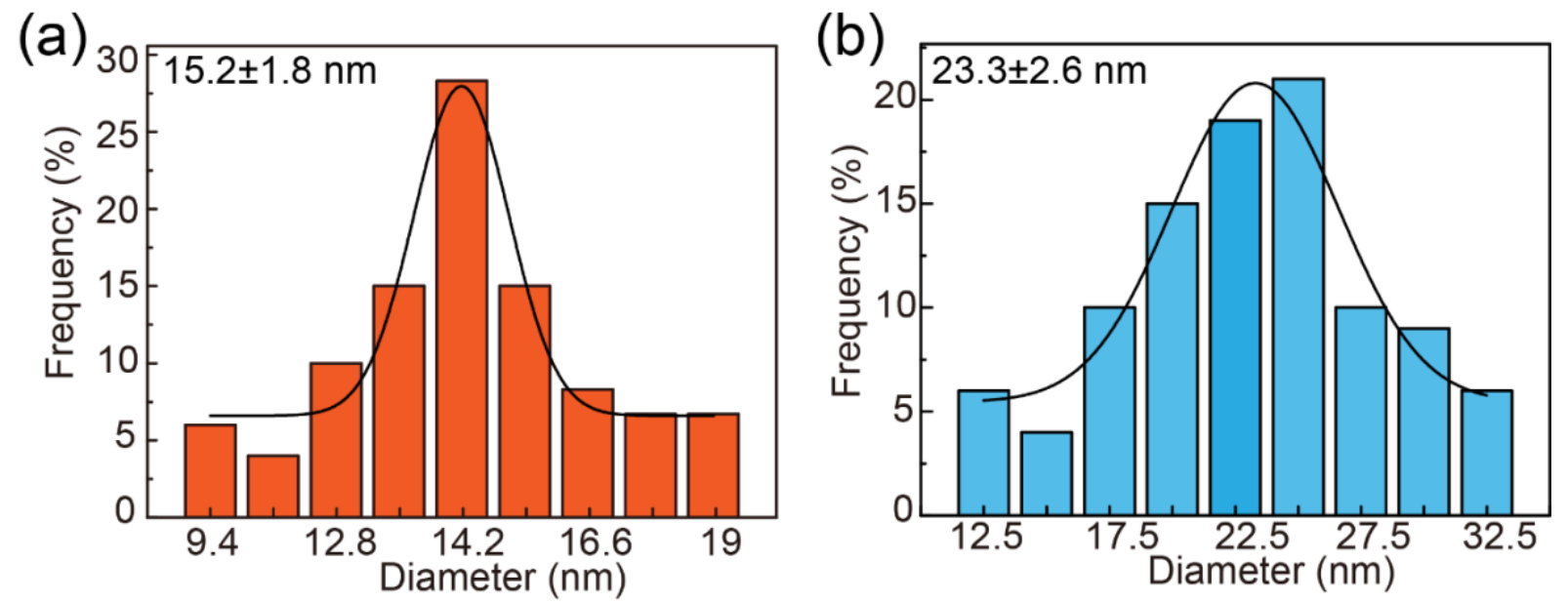

Figure S20. Size distributions of (a) Bi NPs/TAPP-PC and (b) Bi NPs/PC after durability tests. 
Table S1. Elemental contents calculated from XPS results.

\begin{tabular}{ccccc}
\hline \multirow{2}{*}{ Sample } & \multicolumn{4}{c}{ Element content (atomic \%) } \\
\cline { 2 - 5 } PC & $\mathrm{C}$ & $\mathrm{N}$ & $\mathrm{O}$ & $\mathrm{Bi}$ \\
\hline TAPP-PC & 92.91 & 0 & 7.09 & 0 \\
Bi NPs/PC & 89.83 & 1.55 & 8.62 & 0 \\
Bi NPs/TAPP-PC & 90.08 & 0 & 7.54 & 2.38 \\
\hline
\end{tabular}

Table S2. Electrochemical performance comparison of the recently reported Bi-base catalysts for $\mathrm{HCOOH}$ (or formate) production from ECR.

\begin{tabular}{|c|c|c|c|c|c|}
\hline Catalysts & Potential (V) & $\begin{array}{c}\text { FE }_{\text {HCCO }}{ }^{-} \\
(\%)\end{array}$ & $\begin{array}{c}j_{\mathrm{HCOO}^{-}} \\
\left(\mathbf{m A ~ c m}^{-2}\right)\end{array}$ & $\begin{array}{c}\text { Durability } \\
(\text { FE }>80 \% \text { h) }\end{array}$ & Reference \\
\hline $\begin{array}{c}\text { Bi NPs/TAPP- } \\
\text { PC }\end{array}$ & $\begin{array}{c}-0.95 \\
\text { vs. } \text { RHE }\end{array}$ & 92.1 & 55.8 & 20 & This work \\
\hline $\begin{array}{c}\mathrm{Bi} \\
\text { NPs@MWCN } \\
\text { Ts }\end{array}$ & $\begin{array}{c}-1.5 \\
\text { vs. SCE }\end{array}$ & 95.2 & 12.4 & 10 & $\begin{array}{c}\text { ACS } \\
\text { Sustainable } \\
\text { Chem. Eng., } \\
\text { 2020, 8, } 4871\end{array}$ \\
\hline $\begin{array}{l}\text { Ultrathin } \mathrm{Bi} \\
\text { nanosheets }\end{array}$ & $\begin{array}{c}-1.5 \\
\text { vs. SCE }\end{array}$ & 90 & 11 & 10 & $\begin{array}{c}\text { Nat. Commun., } \\
2018,9,1320\end{array}$ \\
\hline
\end{tabular}




\begin{tabular}{|c|c|c|c|c|c|}
\hline Defect rich Bi & $\begin{array}{c}-0.75 \\
\text { vs. RHE }\end{array}$ & 84 & 5 & 24 & $\begin{array}{c}\text { J. Mater. } \\
\text { Chem. A., } \\
2018,6,4714\end{array}$ \\
\hline $\mathrm{BiO}_{\mathrm{x}} / \mathrm{C}$ & $\begin{array}{l}\quad-1.51 \\
\text { vs. } \mathrm{Ag} / \mathrm{AgCl}\end{array}$ & 95.9 & 12.5 & - & $\begin{array}{l}\text { ACS Catal., } \\
2018,8,931\end{array}$ \\
\hline $\mathrm{Bi}_{2} \mathrm{O}_{3}-\mathrm{NGQDs}$ & $\begin{array}{c}-0.87 \\
\text { vs. RHE }\end{array}$ & 98 & 16.6 & 14.5 & $\begin{array}{l}\text { ACS Catal., } \\
2018,8,9926\end{array}$ \\
\hline $\mathrm{Bi} / \mathrm{rGO}$ & $\begin{array}{c}-0.80 \\
\text { vs. RHE }\end{array}$ & 98 & 2 & 15 & $\begin{array}{c}\text { Angew. Chem. } \\
\text { Int. Ed., 2018, } \\
57,12790\end{array}$ \\
\hline $\begin{array}{c}\text { Exfoliated Bi } \\
\text { nanosheets }\end{array}$ & $\begin{array}{c}-1.1 \\
\text { vs. RHE }\end{array}$ & 86 & 16.5 & 10 & $\begin{array}{l}\text { Nano energy, } \\
2018,53,808\end{array}$ \\
\hline Bi-PVP/CC600 & $\begin{array}{c}-0.83 \\
\text { vs. RHE }\end{array}$ & 86 & 54 & 40 & $\begin{array}{l}\text { Appl. Catal., } \\
\text { B, 2021, 284, } \\
\quad 119723\end{array}$ \\
\hline $\mathrm{Bi}_{2} \mathrm{O}_{3} @ \mathrm{C}$ & $\begin{array}{c}-0.9 \\
\text { vs. RHE }\end{array}$ & 93 & 12 & 10 & $\begin{array}{c}\text { Angew. Chem. } \\
\text { Int. Ed., 2020, } \\
59,10807\end{array}$ \\
\hline Bi NTs & $\begin{array}{c}-1.1 \\
\text { vs. RHE }\end{array}$ & 97 & 39.4 & 10 & $\begin{array}{c}\text { ACS Catal., } \\
2020,10,358\end{array}$ \\
\hline $\begin{array}{c}\mathrm{Bi}_{2} \mathrm{O}_{3} \mathrm{NSs} @ \mathrm{M} \\
\mathrm{CCM}\end{array}$ & $\begin{array}{c}-1.256 \\
\text { vs. RHE }\end{array}$ & 93.8 & 17.7 & 12 & $\begin{array}{c}\text { Angew. Chem. } \\
\text { Int. Ed., 2019, } \\
58,13828\end{array}$ \\
\hline $\begin{array}{l}\text { Nanotube- } \\
\text { derived Bi }\end{array}$ & $\begin{array}{c}-1.05 \\
\text { vs. RHE }\end{array}$ & $\sim 100$ & 60 & 5.5 & $\begin{array}{l}\text { Nat. Commun., } \\
2019,10,2807\end{array}$ \\
\hline
\end{tabular}

